\title{
Un modelo multifractal simplificado para flujos de tráfico en redes de computadoras de alta velocidad
}

\section{A simplified multifractal model for traffic flows in high-speed computer networks}

\author{
Ginno Millán Naveas ${ }^{1} \quad$ Enrique San Juan Urrutia ${ }^{2}$ \\ Recibido 26 de septiembre de 2012, aceptado 23 de abril de 2013 \\ Received: September 26, $2012 \quad$ Accepted: April 23, 2013
}

\begin{abstract}
RESUMEN
Este artículo propone un nuevo modelo multifractal, con el ánimo de proveer una posible explicación al fenómeno de localidad que aparece en la estimación del exponente de Hurst en series temporales estacionarias de segundo orden, representativas de los flujos de tráfico autosimilares en las actuales redes de computadoras de alta velocidad. Analíticamente se demuestra que este fenómeno se presenta cuando los flujos se componen de diversos tipos de tráficos con diferentes exponentes de Hurst.
\end{abstract}

Palabras clave: Autosimilitud, exponente de Hurst $(H)$, fenómeno de localidad, modelado de tráfico, multifractales, redes de computadoras de alta velocidad.

\begin{abstract}
This paper proposes a new multifractal model, with the aim to provide a possible explanation to the locality phenomena that appears in the estimation of Hurst exponent in stationary second order temporal series, representing the self-similar traffic flows in high-speed computer networks. Analytically it is shown that this phenomenon occurs if the network flow consists of several components whit different Hurst exponents.

Keywords: Self-similarity, Hurst exponent (H), locality phenomena, traffic modeling, multifractals, highspeed computer networks.
\end{abstract}

\section{INTRODUCCIÓN}

Las propiedades que evidencian la naturaleza de origen fractal de los flujos de tráfico en las redes de computadoras de alta velocidad son un tema que ha sido ampliamente estudiado y reportado en la literatura durante las últimas dos décadas, constituyendo una opinión generalizada el hecho de que su comportamiento dinámico reescalado debe ser cuidadosamente atendido en los análisis de rendimiento y control. Existen, de esta forma, numerosas explicaciones y modelos que intentan responder a este origen [1-3].

Por otra parte, reconociendo que las localidades de un proceso fractal solo pueden ser analizadas desde la óptica del análisis multifractal, dada su construcción a partir de cascadas multiplicativas [4] que aseguran una caracterización más exacta producto del análisis a altas frecuencias [5], se acepta que los flujos de tráfico presentes en las actuales redes de computadoras de alta velocidad poseen naturaleza multifractal, lo cual origina un nuevo modelo de flujos que pretende explicar el fenómeno de localidad presente en la estimación del exponente de Hurst [3, 5].

A partir de los resultados obtenidos mediante el uso de simulaciones computacionales en lenguaje MATLAB, se infiere que el modelo contribuye al conocimiento de la dinámica real del tráfico en las actuales redes de computadoras de alta velocidad, y

\footnotetext{
1 Escuela de Ingeniería. Universidad Católica del Norte. Larrondo 1281. Coquimbo, Chile. E-mail: gmillan@ucn.cl

2 Departamento de Ingeniería Eléctrica. Universidad de Santiago de Chile. Avenida Ecuador 3519, Estación Central. Santiago, Chile. E-mail: enrique.sanjuan@usach.cl
} 
puede ser utilizado para simular de manera simple flujos de tráfico realistas.

\section{SOBRE EL TRÁFICO AUTOSIMILAR Y EL TRÁFICO MULTIFRACTAL}

En las redes de computadoras los flujos de tráfico se consideran a menudo representados mediante un proceso autosimilar $Y(k)$ que satisface [6]

$$
Y(k)={ }_{d} a^{-H} Y(a k), \quad \forall a>0, k \geq 0
$$

donde $=_{d}$ denota igualdad de distribuciones finito dimensionales y $H \in(0,1)$ al exponente de Hurst del proceso estocástico autosimilar $(H-S S) Y(k)$.

Un proceso $H$-SS con incrementos estacionarios descrito en términos del comportamiento de sus agregaciones se obtiene de la multiplexación de incrementos $X(k)=Y(k+1)-Y(k)$ sobre bloques no superpuestos de tamaño $n$ según

$$
X^{(n)}(k)=\sum_{j=0}^{n-1} X(k n-j), \quad k \in \mathbb{Z}, n \in \mathbb{N}
$$

El proceso resultante tiene distribuciones finito dimensionales similares a $X(k)$. Específicamente para cada $n$ se tiene que

$$
X^{(n)}(k)={ }_{d} n^{H} X(k), n \in \mathbb{N}
$$

El proceso estacionario $X(k)$ que cumple con (3) se llama proceso estacionario autosimilar $H$-SSS con exponente de Hurst $H$. Un típico ejemplo es el Ruido Fraccional Gaussiano (FGN) dado por $X(k)=B^{(H)}(k+1)-B^{(H)}(k)$; que es el único proceso gaussiano $H$-SSS conocido [5].

Existen varias formas de estudiar las propiedades estadísticas de $X^{(n)}(k)$. En [5] se consideran los cumulantes de las series agregadas, los cuales se definen en términos de los coeficientes de Taylor de la función generatriz de cumulantes

$g(t)=\log \mathrm{E}\left(e^{t X(k)}\right)=\sum_{m=1}^{\infty} t^{m} m !^{-1} \operatorname{cum}_{m} X(k)$

donde $\operatorname{cum}_{m} X(k)=g^{(m)}(0)$. En [7] se demuestra que los cumulantes de $m$-ésimo orden de un proceso agregado $H$-SSS escalan según una ley de potencia dada por

$$
\operatorname{cum}_{m} X^{(n)}(k)=n^{m H_{S}} \operatorname{cum}_{m} X(k)
$$

Si un proceso estacionario cumple con (5) para todo $n, m \in \mathbb{N}$, entonces el logaritmo del módulo de $\operatorname{cum}_{m} X^{(n)}(k)$ se comporta de forma tal que sus valores escalan linealmente con $\operatorname{los}$ de $\log n$, con coeficientes $m H_{S}$ que son función lineal de $m$. En otras palabras $m H_{S}=m H(m)$, es decir,

$$
\operatorname{cum}_{m} X^{(n)}(k)=n^{m H(m)} \operatorname{cum}_{m} X(k)
$$

En [5] se demuestra que una generalización de un proceso autosimilar a un proceso multifractal viene dada como sigue. Un proceso estacionario $X(k), k$ $\in \mathbb{Z}$, es un proceso multifractal, si

$$
\log \left|\operatorname{cum}_{m} X^{(n)}(k)\right|=m H(m) \log n+c(m)
$$

para todo $m, n \in \mathbb{N}$, permitiendo que el exponente $H$ varíe con el orden $m$.

La forma general para $m H(m)$ está dada por

$$
m H(m)=\alpha m+\beta
$$

que corresponde al modelo fractal lineal, donde los coeficientes $\alpha$ y $\beta$ se determinan (interpolan) directamente durante el ajuste de los cumulantes. En [8-9] se demuestra que el único proceso conocido de este tipo tiene la forma

$$
m H(m)=m+2\left(H_{U}-1\right)
$$

y es llamado proceso unifractal.

En [5] se compara el modelo unifractal con el modelo autosimilar utilizando flujos empíricos. El análisis de las Figuras 3 y 4 de [5] coloca de manifiesto que ambos modelos son capaces de capturar las principales tendencias de los flujos reales en la estimación de $H$, pero además, que ninguno de ellos armoniza perfectamente con el fenómeno de localidad del exponente de Hurst.

La Figura 1 ilustra el fenómeno de localidad del exponente de Hurst. Se observa que la pendiente 
de la curva de ajuste cruza de un valor pequeño a uno notablemente mayor. Por lo tanto la curva se compone de tres partes: un segmento lineal con una pendiente suave cuando $\log n$ es pequeño, una pendiente intermedia, y un segmento lineal con una pendiente más pronunciada cuando $\log n$ es grande.

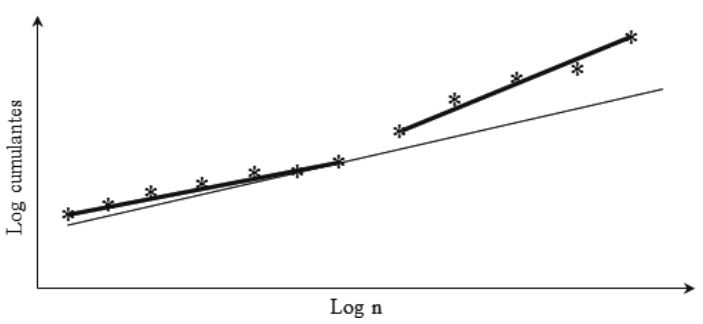

Figura 1. Ilustración del fenómeno de localidad del exponente de Hurst.

\section{UN MODELO MULTIFRACTAL PARA EL FENÓMENO DE LOCALIDAD}

En la referencia [10] se reporta que el análisis de componentes principales (PCA) del espectro de valores propios que resulta de mezclar señales de movimiento browniano fraccional con diferentes exponentes de Hurst, produce un comportamiento biescalar. Este preciso hecho es el que motiva el planteamiento de un modelo de flujo multifractal capaz de reproducir el fenómeno de localidad del exponente de Hurst.

Suponiendo un proceso de flujo de tráfico de red $W(k)$, compuesto por la adición de dos procesos autosimilares independientes; $X_{1}(k)$ y $X_{2}(k)$, con exponentes de Hurst $H_{1}$ y $H_{2}$, respectivamente, es decir

$$
W(k)=\alpha_{1} X_{1}(k)+\alpha_{2} X_{2}(k)
$$

donde $\operatorname{Var}\left\{X_{1}\right\}=\operatorname{Var}\left\{X_{2}\right\}$ y los coeficientes de escala $\alpha_{1}$ y $\alpha_{2}\left(\alpha_{1}, \alpha_{2}>0\right)$ controlan la varianza de las componentes de (10). Además, sin pérdida de generalidad que $H_{1}<H_{2}$. Al igual que para el caso de (2), se puede definir el proceso agregado $Z^{(n)}(k)$ mediante la multiplexación de incrementos $Z(k)=W(k+1)-W(k)$ considerando bloques no superpuestos de tamaño $n$ como

$$
Z^{(n)}(k)=\sum_{j=0}^{n-1} X(k n-j), \quad k \in \mathbb{Z}, n \in \mathbb{N}
$$

Por independencia, para los modelos autosimilar y unifractal se verifica que

$$
\begin{aligned}
& \left|\operatorname{cum}_{m} Z^{(n)}(k)\right|= \\
& \left|\operatorname{cum}_{m}\left(\alpha_{1} X_{1}^{(n)}(k)+\alpha_{2} X_{2}^{(n)}(k)\right)\right|
\end{aligned}
$$

es decir,

$$
\begin{aligned}
& \left|\operatorname{cum}_{m} Z^{(n)}(k)\right|= \\
& c_{1}(m) n^{n H_{1}(m)}+c_{2}(m) n^{n H_{2}(m)}= \\
& c_{1}(m) n^{m+2\left(H_{1}(m)-1\right)}+c_{2}(m) n^{m+2\left(H_{2}(m)-1\right)}
\end{aligned}
$$

con $c_{1}(m)$ y $c_{2}(m)$ parcialmente determinados por los coeficientes $\alpha_{1}$ y $\alpha_{2}$, respectivamente.

Luego, si $c_{1}(m)>c_{2}(m)$ existe una única solución positiva $n^{*}$ (que no es necesariamente entera) para la ecuación en términos de la variable $n$

$$
c_{1}(m) n^{m+2\left(H_{1}(m)-1\right)}=c_{2}(m) n^{m+2\left(H_{2}(m)-1\right)}
$$

Así, resulta fácil verificar que

$$
\begin{aligned}
& \left|\operatorname{cum}_{m} Z^{(n)}(k)\right|= \\
& \left\{\begin{array}{cc}
c_{1}(m) n^{m+2\left(H_{1}(m)-1\right)}, & \text { para } n \ll n^{*} \\
c_{2}(m) n^{m+2\left(H_{2}(m)-1\right)}, & \text { para } n \gg n^{*}
\end{array}\right.
\end{aligned}
$$

Para cada $m$ el diagrama en escala logarítmica del cumulante de $m$-ésimo orden de $Z^{(n)}(k)$ consiste en tres segmentos: un primer segmento lineal con pendiente $m+2\left(H_{1}-1\right)$ cuando $n$ es pequeño; una sección de transición intermedia (a menudo muy corta) y, finalmente, un segmento lineal con pendiente $m+2\left(H_{2}-1\right)$ cuando $n$ es grande. Por lo tanto se coloca en evidencia el fenómeno de localidad del exponente de Hurst.

La Figura 2 expone el fenómeno de localidad del exponente de Hurst para una serie FGN generada espectralmente [11] con $H=0,6$, mientras que la Figura 3 lo hace para una serie FGN con $H=0,8$ también generada espectralmente. Finalmente, la Figura 4 muestra el comportamiento conjunto de ambas series considerando $\lambda_{1}=\lambda_{2}$ en (13). 


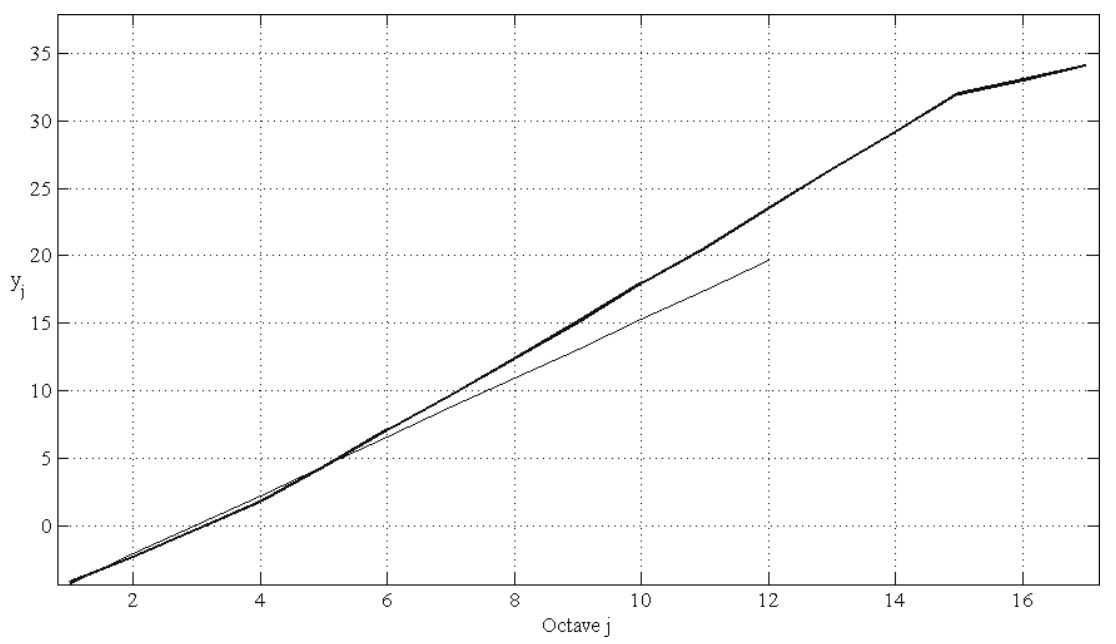

Figura 2. Fenómeno de localidad en una serie FGN con $H=0,6$ generada espectralmente.

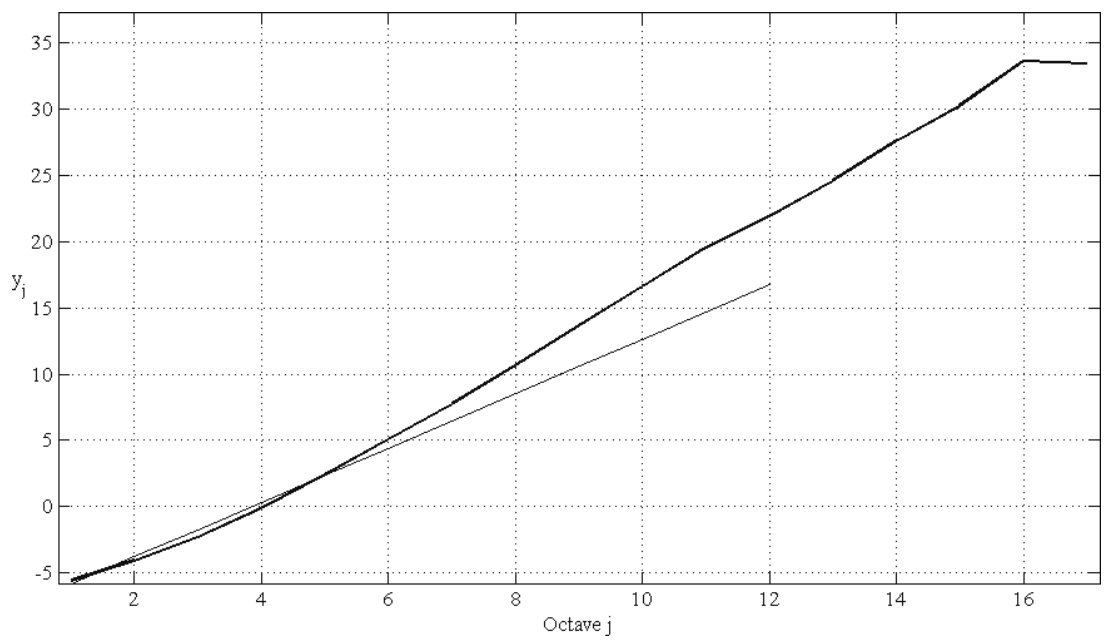

Figura 3. Fenómeno de localidad en una serie FGN con $H=0,8$ generada espectralmente.

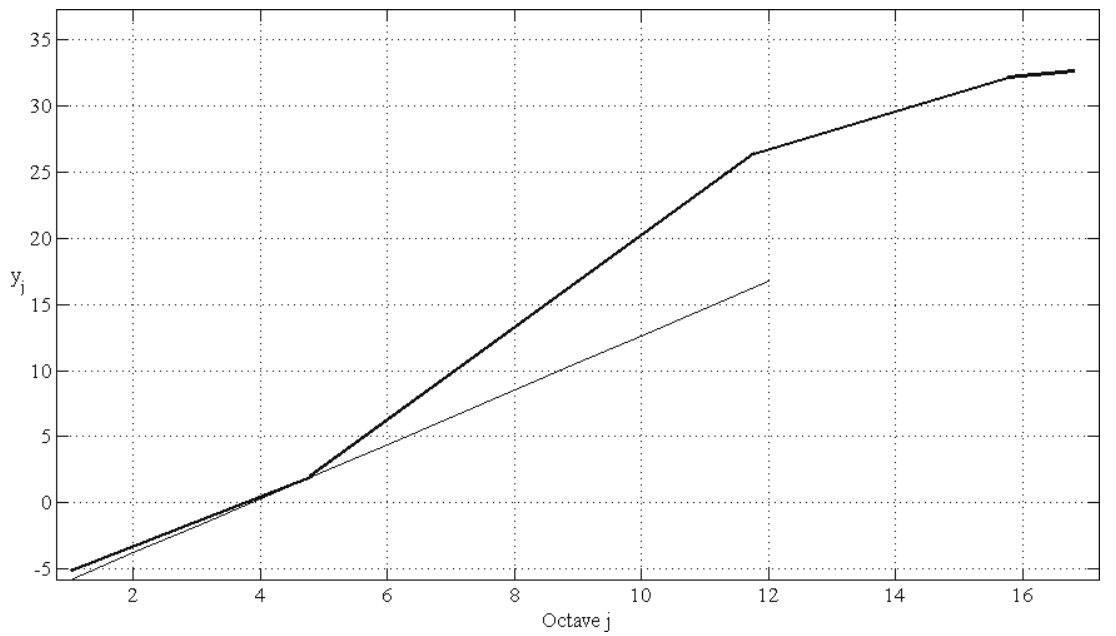

Figura 4. Fenómeno de localidad observado en el comportamiento conjunto de las series anteriores. 
Con todo lo anterior se demuestra de una manera simple que si un proceso de flujo de tráfico se forma a partir de dos componentes autosimilares aditivos independientes con exponentes de Hurst diferentes, entonces se observa el fenómeno de localidad en la estimación del exponente de Hurst empleando cumulantes. No resulta difícil ver que este modelo puede extenderse al caso de más de dos componentes con similares resultados.

\section{CONCLUSIONES}

Resulta interesante observar que la inconsistencia teórica de un único valor del exponente de Hurst y el fenómeno de localidad pueden ser resueltos empleando un simple modelo que adiciona series temporales autosimilares de segundo orden. Por otra parte, es posible generar varios procesos con diferentes exponentes de Hurst, empleando para ello cualquiera de los métodos existentes para la generación de series autosimilares de segundo orden y luego adicionarlos. Esto está de acuerdo con la conclusión de [12], que establece que es necesario multiplexar diversas fuentes de tráfico para generar simulaciones más apropiadas para los flujos de tráfico.

La mayoría de los modelos anteriores que tratan los orígenes de la autosimilitud de los flujos de tráfico en las redes de alta velocidad pueden ser adoptados, desde la perspectiva de su aplicación al modelo propuesto, con el objetivo de recalcar la coexistencia en los flujos reales de múltiples componentes autosimilares heterogéneos. Puede así afirmarse que si en el modelo On/Off de [13] se supone la existencia de dos valores diferentes para los exponentes de Pareto que controlan las distribuciones los periodos On y los periodos Off de las fuentes, entonces el proceso de flujo final agregado presentará dos componentes diferentes en términos de sus exponentes de Hurst y, por lo tanto, dará cuenta de la existencia del fenómeno de localidad.

En general, para redes de grandes coberturas, es decir, con estructuras muy complejas, el canal de datos es compartido por múltiples fuentes en una forma que puede considerarse aproximadamente independiente y aditiva. Debido a la diversidad de las fuentes y los mecanismos de transferencia, el flujo que ingresa proveniente de cada fuente posee diferentes exponentes de Hurst. Esto lleva a plantear la siguiente conjetura: es más probable observar el fenómeno de localidad en flujos de tráfico de redes troncales que locales.

\section{AGRADECIMIENTOS}

Se agradece al programa de formación de capital humano avanzado CONICYT 2012, por el apoyo brindado al autor G. Millán por medio de la beca para estudios de doctorados en Chile.

\section{REFERENCIAS}

[1] G. Millán, H. Kaschel and G. Lefranc. "Discussion of the analysis of self-similar teletraffic with long-range dependence (LRD) at the network layer level". Int. J. Comput. Commun. Vol. V, Issue 5, pp. 799-812. December, 2010.

[2] Y.-D. Chen, L. Li, Y. Zhang and J.-M. Hu. "Fluctuations and pseudo long range dependence in network flows: a non-stationary Poisson process model". Chinese Physics B. Vol. 18, Issue 4, pp. 1373-1379. April, 2009.

[3] G. Millán, H. Kaschel and G. Lefranc. "A simple model for the generation of LRD self-similar traffic using piecewise affine chaotic one-dimensional maps". Stud. Inform. Control. Vol. 19, Issue 1, pp. 67-78. March, 2010.

[4] J.G. Evertsz and B.B. Mandelbrot. "Multifractal measures". In Chaos and Fractals, H.-O. Peitgen, H. Jürgens, and D. Saupe, Eds. 1st Ed. Springer-Verlag, New York, Appendix B, pp. 849-881. 1992.

[5] G. Terdik and T. Gyires. "Lévy flights and fractal modeling of Internet traffic". IEEE/ ACM Trans. Netw. Vol. 17, Issue 1, pp. 120129. February, 2009.

[6] O.I. Sheluhin, S.M. Smolskiy and A.V. Osin. "Self-Similar Processes in Telecommunications". Wiley. UK. 2007.

[7] G. Terdik. "Bilinear stochastic models and related problems of nonlinear time series analysis: a frequency approach". Lectures Notes in Statistics. Vol. 142. Springer-Verlag. New York. 1999.

[8] E. Iglói and G. Terdik. "Superposition of diffusions with linear generator and its 
multifractal limit process". ESAIM-Probab. Stat. Vol. 7, pp. 23-88. May, 2003.

[9] S. Molnár and G. Terdik. "A general fractal model of Internet traffic". In Proc. 26th Annu. IEEE Conf. Local Computer Networks. Tampa, FL, pp. 492-499. 2001.

[10] L. Li, J. Hu, Y. Chen and Y. Zhang. "PCA based Hurst exponent for $\mathrm{fBm}$ signals under disturbances". IEEE Trans. Signal Process. Vol. 57, Issue 7, pp. 2840-2846. July, 2009.

[11] P. Abry, P. Flandrin, M.S. Taqqu and D. Veitch. "Self-similarity and long-range dependence through the wavelets lens". In Theory and Applications of Long-Range Dependence, P.
Doukhan, G. Oppenheim and M. S. Taqqu, Eds. Birkhäuser, Boston, MA, pp. 527-556. 2003.

[12] G. Horn, A. Kvalbein, J. Blomskøld and E. Nilsen. "An empirical comparison of generators for self-similar simulated traffic". Perform. Evaluation. Vol. 64, Issue 2, pp. 162190. February, 2007.

[13] W. Willinger, M.S. Taqqu, R. Sherman and D.V. Wilson. "Self-similarity through highvariability: statistical analysis of Ethernet LAN traffic at the source level". IEEE/ ACM Trans. Netw. Vol. 5, Issue 1, pp. 71-86. February, 1997. 